\title{
AN APPLICATION OF CODING THEORY TO ESTIMATING DAVENPORT CONSTANTS
}

\author{
ALAIN PLAGNE AND WOLFGANG A. SCHMID
}

\begin{abstract}
We investigate a certain well-established generalization of the Davenport constant. For $j$ a positive integer (the case $j=1$, is the classical one) and a finite Abelian group $(G,+, 0)$, the invariant $\mathrm{D}_{j}(G)$ is defined as the smallest $\ell$ such that each sequence over $G$ of length at least $\ell$ has $j$ disjoint non-empty zero-sum subsequences. We investigate these quantities for elementary 2-groups of large rank (relative to $j$ ). Using tools from coding theory, we give fairly precise estimates for these quantities. We use our results to give improved bounds for the classical Davenport constant of certain groups.
\end{abstract}

\section{INTRODUCTION}

For a given Abelian group $(G,+, 0)$, the Davenport constant, denoted $\mathrm{D}(G)$, is defined as the smallest integer $\ell$ such that each sequence over $G$ of length at least $\ell$ has a non-empty zero-sum subsequence, i.e. the sum of its terms is 0. Equivalently, $\mathrm{D}(G)$ is the maximal length of a minimal zero-sum sequence over $G$, i.e. the maximal length of a sequence of elements of $G$ summing to 0 and with no proper subsequence summing to 0 .

It is considered as a central object in combinatorial number theory since Davenport popularized it in the 60's (as reported in [25]), notably for its link with algebraic number theory, see e.g. 17] or [16. In fact it seems that the first paper that deals with this invariant was written by Rogers [28, who himself attributes the paternity of the problem to Sudler.

This invariant has become the prototype of algebraic invariants of combinatorial flavour. Since the 60 's, the theory of these invariants has highly developed in several directions; see for instance the survey article [15] or Chapters 5, 6, and 7 of [17].

Let $G$ be written, as is always possible, as a direct sum of cyclic groups $G \cong$ $C_{n_{1}} \oplus \cdots \oplus C_{n_{r}}$ with integers $1<n_{1}|\cdots| n_{r}$ ( $r$ denotes the rank of $G$, and $n_{r}$ the exponent, except for $r=0$ where the exponent is 1$)$. Then, the basic lower bound for Davenport constant is

$$
\mathrm{D}(G) \geq 1+\sum_{i=1}^{r}\left(n_{i}-1\right)
$$

to see this, note that a sequence containing only, for each cyclic component $C_{n_{i}}$ $(1 \leq i \leq n)$, one generating element $n_{i}-1$ times, has no non-empty zero-sum subsequence.

It is known that for groups of rank at most two and for $p$-groups ( $p$, a prime), inequality (10) is in fact an equality; this was obtained independently in [11] and

2010 MSC: 11B30, 11B75, 11P70, 94B05, 94B65

W.S. is supported by the Austrian Science Fund (FWF): J 2907-N18. 
25, 26. For groups of rank at least four, equality is definitely not the rule (see [1, 11, 18]). In the case of groups of rank three, it is conjectured that equality always holds but this conjecture is wide open (see [15]).

Concerning upper bounds, the best general result is the following

$$
\mathrm{D}(G) \leq \exp (G)\left(1+\log \frac{|G|}{\exp (G)}\right)
$$

proved in [12, 23].

In view of the depicted situation, it appears that it is generically very difficult to determine the Davenport constant of a group (of rank at least three). In particular, despite various works related to the Davenport constant over the years, its actual value was only determined for a few additional - beyond the ones known since the end of the 60's - families of groups; see 3 for a recent contribution.

The $j$-wise Davenport constants are defined depending on a positive integer $j$. We define $\mathrm{D}_{j}(G)$ to be the smallest $\ell$ such that each sequence over $G$ of length at least $\ell$ has $j$ disjoint non-empty zero-sum subsequences. Equivalently, the maximal length of a zero-sum sequence over $G$ that cannot be decomposed into $j+1$ nonempty zero-sum sequences. Evidently $\mathrm{D}_{1}(G)=\mathrm{D}(G)$ and for any positive $j$ one has $\mathrm{D}_{j}(G) \leq j \mathrm{D}(G)$.

This variant of the Davenport constant was introduced by Halter-Koch [19] in order to determine the order of magnitude of the counting function of the set of algebraic integers of some number field that are not divisible by a product of $j+1$ irreducible algebraic integers of this number field. It is

$$
\frac{x}{\log x}(\log \log x)^{\mathrm{D}_{j}(G)-1}
$$

where $G$ denotes the ideal class group of the number field considered. Moreover, knowledge of these invariants is useful in investigations of the Davenport constant itself (cf. below for details and recall e.g. that a considerable part of [3], determining $\mathrm{D}\left(C_{3}^{2} \oplus C_{3 n}\right)$, is devoted to determining $\mathrm{D}_{j}\left(C_{3}^{3}\right)$ and closely related problems).

The case of cyclic groups is the simplest one since then it is easy to see that $j n-1<\mathrm{D}_{j}\left(C_{n}\right) \leq j \mathrm{D}\left(C_{n}\right)=j n$ (for the lower bound, simply take a generating element repeated that number of times). The case of groups of rank two is also known [19, as well as the case of certain closely related groups [17, Section 6.1]. But in general computing (even bounding) $\mathrm{D}_{j}(G)$ is quite more complicated than for $\mathrm{D}(G)$, in particular for (elementary) $p$-groups. For example, $\mathrm{D}_{j}(G)$ for all $j$, is only known for the following elementary $p$-groups of rank greater than two: $C_{2}^{3}$, $C_{2}^{4}, C_{2}^{5}$, and $C_{3}^{3}$ (see [8, 14, 3]).

The main difference is that it seems that the types of arguments used to determine $\mathrm{D}(G)$ for $p$-groups cannot be applied. At first this might seem surprising, however recall that the same phenomenon is encountered for the invariant $\eta(G)$ (cf. Section 2) and other closely related invariants such as the Erdös-Ginzburg-Ziv constant. In particular, the difficulty of the problem seems to increase with the rank of the groups considered (to be precise, the key quantity is the size of the rank relative to the exponent), as then $\mathrm{D}(G)$ and $\eta(G)$ are far apart (cf. Section 2 for the relevance of this fact).

In this paper and from now on, we focus on the case of elementary 2-groups with large rank. The reason for this is two-fold. On the one hand, it is an interesting case; the rank is 'maximal' relative to the exponent. On the other hand, the special 
nature of the group allows certain arguments that fail in more general situations; for instance recall that to show $\eta\left(C_{2}^{r}\right)=2^{r}$ is almost trivial, yet the problem of determining $\eta\left(C_{p}^{r}\right)$ for any (fixed) odd prime $p$ and arbitrary $r$ is wide open (it is even open, which order of magnitude is to be expected).

While it is easy to determine that $\mathrm{D}_{1}\left(C_{2}^{r}\right)=r+1$ - we effectively consider a vector space over a field with a unique non-zero element, and having no non-empty zero-sum subsequences is thus equivalent to linear independence - the situation becomes more complicated for larger values of $j$. We observe that

$$
r<\mathrm{D}_{1}\left(C_{2}^{r}\right) \leq \mathrm{D}_{j}\left(C_{2}^{r}\right) \leq j \mathrm{D}_{1}\left(C_{2}^{r}\right) \leq j(r+1)
$$

Thus, for a fixed positive integer $j$, the sequence $\left(\mathrm{D}_{j}\left(C_{2}^{r}\right)\right)_{r \in \mathbb{N}}$ has to grow linearly. Our main aim here is to make this statement more precise and to study the following relevant quantity

$$
\frac{\mathrm{D}_{j}\left(C_{2}^{r}\right)}{r},
$$

of which we examine the asymptotic behaviour when $r$ becomes large. The quantities

$$
\alpha_{j}=\liminf _{r \rightarrow+\infty} \frac{\mathrm{D}_{j}\left(C_{2}^{r}\right)}{r} \quad \text { and } \quad \beta_{j}=\limsup _{r \rightarrow+\infty} \frac{\mathrm{D}_{j}\left(C_{2}^{r}\right)}{r}
$$

will be considered and investigated. By the above crude reasoning we only get that $1 \leq \alpha_{j} \leq \beta_{j} \leq j$. Our first theorem, which gives explicit estimates for small values of $j$, improves on this. It is a generalization of results by Komlós (lower bound, quoted in [24, 5]) and by Katona and Srivastava (upper bound) [20] for the case $j=2$ that we recall for the sake of completeness in the statement of the theorem.

Theorem 1. For each sufficiently large integer $r$ we have

$1.261 r \leq \mathrm{D}_{2}\left(C_{2}^{r}\right) \leq 1.396 r$,
$1.500 r \leq \mathrm{D}_{3}\left(C_{2}^{r}\right) \leq 1.771 r$,
$1.723 r \leq \mathrm{D}_{4}\left(C_{2}^{r}\right) \leq 2.131 r$,
$1.934 r \leq \mathrm{D}_{5}\left(C_{2}^{r}\right) \leq 2.478 r$
$2.137 r \leq \mathrm{D}_{6}\left(C_{2}^{r}\right) \leq 2.815 r$,
$2.333 r \leq \mathrm{D}_{7}\left(C_{2}^{r}\right) \leq 3.143 r$,
$2.523 r \leq \mathrm{D}_{8}\left(C_{2}^{r}\right) \leq 3.464 r$,
$2.709 r \leq \mathrm{D}_{9}\left(C_{2}^{r}\right) \leq 3.778 r$,
$2.890 r \leq \mathrm{D}_{10}\left(C_{2}^{r}\right) \leq 4.087 r$.

As will be apparent from our arguments, having our method at hand, to expand this list further is merely a computational effort.

Notice that the question whether $\alpha_{j}=\beta_{j}$ (independently of the value that this constant would take) seems not obvious.

After the study of small values for $j$, we turn our attention to the case of large $j$ 's. Although we are unable to prove that $\alpha_{j}$ and $\beta_{j}$ are equal, which seems conceivable, we show that they at least grow at the same speed and more precisely determine their order of magnitude.

Theorem 2. When $j$ tends to infinity, we have the following:

$$
\log 2\left(\frac{j}{\log j}\right) \lesssim \liminf _{r \rightarrow+\infty} \frac{\mathrm{D}_{j}\left(C_{2}^{r}\right)}{r} \leq \limsup _{r \rightarrow+\infty} \frac{\mathrm{D}_{j}\left(C_{2}^{r}\right)}{r} \lesssim 2 \log 2\left(\frac{j}{\log j}\right) .
$$


We believe that the bound for the lim inf is closer to the actual value. A heuristic suggests that the $\lim \sup$ in Theorem 2 is in fact close to $\log 2(j / \log j)$ as well. More precisely, we formulate the following conjecture.

Conjecture 3. For any positive integer $j$, the limit

$$
\gamma_{j}=\lim _{r \rightarrow+\infty} \frac{\mathrm{D}_{j}\left(C_{2}^{r}\right)}{r}
$$

exists and one has

$$
\gamma_{j} \sim \log 2\left(\frac{j}{\log j}\right)
$$

as $j$ tends to infinity.

For results in the converse scenario, that is fixed but arbitrary $r$, and $j$ goes to infinity, see [14.

One of the main reasons for studying $j$-wise Davenport constants is the fact that they are important in obtaining results on the Davenport constant itself. The connection is encoded in the following inequality, due to Delorme, Ordaz, and Quiroz 8 . For a finite Abelian group $G$ and a subgroup $H$ one has

$$
\mathrm{D}(G) \leq \mathrm{D}_{\mathrm{D}(H)}(G / H) .
$$

Among others, this inequality encodes the classical form of the inductive method, originally introduced to determine the Davenport constant for groups of rank two (see [28, 11, 26]).

We can apply our results on the $j$-wise Davenport constants to obtain improved bounds on the Davenport constant for certain types of groups (the 2-rank has to be 'large' relative to the order). We only formulate it explicitly for a quite special type of group, which however, due to its extremal nature, is of relevance in this context.

Corollary 4. When $n$ tends to infinity, we have

$$
\limsup _{r \rightarrow+\infty} \frac{\mathrm{D}\left(C_{2}^{r-1} \oplus C_{2 n}\right)}{r} \lesssim 2 \log 2 \frac{n}{\log n} .
$$

For comparison, the general bound mentioned above yields only

$$
\limsup _{r \rightarrow+\infty} \frac{\mathrm{D}\left(C_{2}^{r-1} \oplus C_{2 n}\right)}{r} \lesssim 2 \log 2 n .
$$

We immediately give the short proof of this result.

Proof. By (2), we get that

$$
\mathrm{D}\left(C_{2}^{r-1} \oplus C_{2 n}\right) \leq \mathrm{D}_{\mathrm{D}\left(C_{n}\right)}\left(C_{2}^{r}\right)=\mathrm{D}_{n}\left(C_{2}^{r}\right) .
$$

By Theorem 2, the claim follows.

We finish this Introduction with outlining the plan of the present article. In Section 2, we explain the methods and the prerequisites we need in the course of this article. In Section 3, we derive the lower bounds of our two results while in Section 4 the upper bounds are proved. Finally, in Section 5 , we discuss the heuristic leading to Conjecture 3. If true, this heuristic would establish at least the asymptotic equivalence of $\alpha_{j}$ and $\beta_{j}$ and imply the second part of Conjecture 3 . 


\section{The methods}

We outline the methods we use to establish our results.

2.1. Zero-sum subsequences of bounded length. Let $G$ be an Abelian group. For $x$ a real number, let $\mathrm{s}_{\leq x}(G)$ denote the smallest element $\ell \in \mathbb{N} \cup\{+\infty\}$ such that each sequence of length at least $\ell$ has a zero-sum subsequence of length at most $x$. Evidently, $\mathbf{s}_{\leq x}(G)=\mathbf{s}_{\leq\lfloor x\rfloor}(G)$, yet for technical reasons it is useful to define $\mathbf{s}_{\leq x}(G)$ for non-integral $x$ as well.

A prominent special case of this definition is $x=\exp (G)$, the resulting invariant is typically denoted by $\eta(G)$; note that for $x<\exp (G)$, one has $\mathrm{s}_{\leq x}(G)=+\infty$. Also, note that for $x \geq \mathrm{D}(G)$ we have $\mathrm{s}_{\leq x}(G)=\mathrm{D}(G)$.

For results on $\mathbf{s}_{\leq x}(G)$, for generic $x$, mainly for elementary 2- and 3-groups, see e.g. [8, 3], and [7] for a very closely related problem (cf. below). For recent results on $\eta(G)$, focusing on lower bounds, see [10, 9].

To determine $\eta(G)$ seems to be a very difficult problem in general. For example, $\eta\left(C_{3}^{6}\right)$ was determined only recently [27, despite the fact that the problem of determining $\eta\left(C_{3}^{r}\right)$ is fairly popular (see [10] for a detailed outline of several problems, and their respective history, that are equivalent to determining $\left.\eta\left(C_{3}^{r}\right)\right)$.

Yet, in the case of elementary 2-groups, to determine both $\eta\left(C_{2}^{r}\right)$ and $\mathrm{D}\left(C_{2}^{r}\right)$ is almost trivial. However, for other values of $x$ even for elementary 2-groups the problem of determining $\mathbf{s}_{\leq x}\left(C_{2}^{r}\right)$ is not at all trivial, namely, it is equivalent to a central problem of coding theory (cf. below).

It is known, in particular by the work of Delorme, Ordaz, and Quiroz [8], that the invariants $\mathrm{s}_{\leq x}(G)$ can be used to derive upper bounds for $\mathrm{D}_{j}(G)$. More specifically, we have (this is Lemma 2.4 in [14])

$$
\mathrm{D}_{j+1}(G) \leq \min _{i \in \mathbb{N}} \max \left\{\mathrm{D}_{j}(G)+i, \mathrm{~s}_{\leq i}(G)-1\right\}
$$

Thus, knowing $\mathrm{D}_{1}(G)=\mathrm{D}(G)$ and the constants $\mathbf{s}_{\leq i}(G)$, or bounds for these constants, one can obtain, recursively applying estimate (3), bounds for $\mathrm{D}_{j}(G)$. Notice however that even exact knowledge of $\mathrm{D}(G)$ and $\mathbf{s}_{\leq i}(G)$ for all $i$ can be insufficient to determine $\mathrm{D}_{j}(G)$ exactly via this method, which in general is not optimal.

2.2. Coding theory enters the picture. We recall that the link between coding theory and combinatorial number theory is not new. For instance, Cohen, Litsyn, and Zémor [4] used coding theoretic bounds in the Sidon problem. The general paper [7] by two of these authors provides a worthwhile introduction to the links between the two problematics. Also, Freeze [13] used coding theory in the present context.

One of the reasons for this connection is the following folkloric lemma [21].

Lemma 5. The minimal distance of a binary linear code $\mathcal{C}$ is equal to the minimal length of a zero-sum subsequence of columns of a parity check matrix of $\mathcal{C}$.

In the case of present interest, Cohen and Zémor [7] pointed out a connection between $\mathbf{s}_{\leq i}\left(C_{2}^{r}\right)$ and coding theory. As the situation at hand is slightly different from the one in that paper, and this connection is central to our investigations, we recall and slightly expand it in some detail.

First, we give a technically useful definition. In the present context, we call a function $f:[0,1] \rightarrow[0,1]$ upper-bounding if it is decreasing (not necessarily 
strictly), continuous and each $[n, k, d]$ code satisfies

$$
\frac{k}{n} \leq f\left(\frac{d}{n}\right)
$$

In other words, upper-bounding functions are the functions intervening in the upper bounds of the rate of a code by a function of its normalized minimal distance.

We call a function asymptotically upper-bounding if it has the same properties as an upper-bounding function, except that the inequality only has to hold for $[n, k, d]$ codes with sufficiently large $n$.

Notice that in both cases, the assumptions on decreasingness and continuity are not restrictive at all since these assumptions are usually fulfilled.

Lemma 6. Let $f$ be an upper-bounding function. Let $d, n$, and $r$ be three positive integers satisfying $2 \leq d \leq n-1$ and

$$
\frac{n-r}{n}>f\left(\frac{d+1}{n}\right)
$$

then

$$
\mathrm{s}_{\leq d}\left(C_{2}^{r}\right) \leq n
$$

Moreover, the same assertion holds true for $f$ an asymptotically upper-bounding function if we impose that $n$ is sufficiently large (depending on $f$ ).

Proof. Let $S=g_{1}, \ldots, g_{n}$ be an arbitrary finite sequence over $C_{2}^{r}$. We shall prove that it contains a zero-sum subsequence of length at most $d$.

It is immediate that $S$ has a zero-sum subsequence of length 1 if and only if 0 occurs in $S$, and that $S$ has a zero-sum subsequence of length 2 if and only if some element occurs at least twice in $S$. Thus, since $d \geq 2$, we may assume that $S$ does neither contain 0 nor an element more than once i.e. we effectively have to study the case of subsets of $C_{2}^{r} \backslash\{0\}$ (and not the one of general sequences in $C_{2}^{r}$ ).

We assert that we may assume that the elements appearing in $S$ generate $C_{2}^{r}$. To see this, note that if $g$ is an element of $C_{2}^{r}$ not contained in the subgroup generated by the elements of $S$, and if $T$ denotes the sequence obtained by appending $g$ to $S$, then each zero-sum subsequence of $T$ is in fact a zero-sum subsequence of $S$. Thus, if $S$ has no zero-sum subsequence of length at most $d$ and the elements of $S$ do not generate $C_{2}^{r}$, then the longer sequence $T$, defined as above, neither has a zero-sum subsequence of length at most $d$. So, it suffices to establish an upper bound on the length of sequences $S$ such that the elements appearing in $S$ generate $C_{2}^{r}$.

We choose some basis of $C_{2}^{n}$. We consider the binary linear code $\mathcal{C} \subset C_{2}^{n}$ of length $n$ whose parity check matrix is $A=\left[g_{1}|\cdots| g_{n}\right] \in \mathcal{M}_{r, n}$ (identify the $g_{i}$ 's with their coordinate vectors with respect to some basis of $C_{2}^{r}$, and consider them as column vectors, and use the just chosen basis of $C_{2}^{n}$ ). Notice that the rank of this matrix is equal to $r$ in view of our assumption that the $g_{i}$ 's generate $C_{2}^{r}$. Let $m$ be the minimal distance of $\mathcal{C}$. By definition, the code $\mathcal{C}$ is an $[n, n-r, m]$ binary linear code. But by assumption since $f$ is upper-bounding and

$$
\frac{n-r}{n}>f\left(\frac{d+1}{n}\right)
$$

an $[n, n-r, d+1]$ code cannot exist. This implies that $m<d+1$, or equivalently $d \geq m$. 
We conclude by applying Lemma 5 which shows that $S$ possesses a zero-sum subsequence of length $m$.

The additional claim for asymptotically upper-bounding functions, is immediate in view of the just given argument.

\section{LOWER BOUNDS}

In this section, we establish the lower bounds for $\mathrm{D}_{j}\left(C_{2}^{r}\right)$, for large $r$, contained in Theorems 1 and 2. Specifically, we prove the following asymptotic lower bound in $r$, which immediately yields both lower bounds (replacing $\log (j+1)$ by $\log j$ is asymptotically, in $j$, irrelevant).

Proposition 7. Let $j$ be a positive integer. Then

$$
\mathrm{D}_{j}\left(C_{2}^{r}\right) \geq \log 2 \frac{j}{\log (j+1)} r
$$

as $r$ tends to infinity.

Notice that the case $j=1$ is essentially trivial, while the case $j=2$ of this result, formulated in the context of coding theory, is attributed to Komlós in [5] and [24]. Indeed, our proof will generalize Komlós' approach (in the form given in [5]). This proof can be seen as probabilistic, yet we prefer to present it via a direct counting argument. Notice that it is non-constructive.

We need the following well-known lemma (see e.g. [21]).

Lemma 8. Let $n$ and $k$ be two positive integers, $n \geq k$. In an $n$-dimensional vector space over a field with 2 elements, the number of $k$-dimensional subspaces is equal to the 2-ary binomial coefficient defined as

$$
\left[\begin{array}{l}
n \\
k
\end{array}\right]=\frac{\left(2^{n}-1\right) \cdots\left(2^{n-k+1}-1\right)}{\left(2^{k}-1\right) \cdots(2-1)} .
$$

Moreover, the number of $k$-dimensional subspaces containing a fixed $j$-dimensional subspace, $k \geq j$, is equal to

$$
\left[\begin{array}{l}
n-j \\
k-j
\end{array}\right]
$$

We can now prove Proposition 7

Proof of Proposition 7. For the entire proof, we fix an arbitrary positive integer $j>1$. As $\mathrm{D}_{1}\left(C_{2}^{r}\right)=\mathrm{D}\left(C_{2}^{r}\right)=r+1$, we can ignore the case $j=1$.

We shall now prove that for each integer $n$ larger than or equal to $r+j$ (this condition is technically convenient later on) and less than $(j \log 2 / \log (j+1)) r$ (notice that, for $r$ large enough, since $j \geq 2$, such $n$ 's always exist), one can find a sequence of cardinality $n$ which does not contain $j$ disjoint zero-sum subsequences. This will prove our result.

To each sequence $S=g_{1}, \ldots, g_{n}$ over $C_{2}^{r}$, with $n \geq r+j$, having the properties that $S$ does neither contain 0 nor an element at least twice, we associate, as described above, an $[n, n-r]$ code (contained in $C_{2}^{n}$, and we fix some basis). This linear code, automatically, has a minimal distance of at least 3. Conversely, any $[n, n-r, d]$ code with $d \geq 3$ can be obtained in this way (cf. [7]).

The following remark is central: the condition that $S$ has $j$ disjoint zero-sum subsequences translates to the condition that the associated code contains $j$ nonzero codewords $c_{1}, \ldots, c_{j}$ such that intersection of the support (the set of indices 
of non-zero coordinates) of $c_{u}$ and $c_{v}$ is empty for all distinct $u, v \in\{1, \ldots, j\}$. A code having this property will be called $j$-inadmissible, otherwise it will be called j-admissible.

We first produce an upper bound on the total number of $[n, n-r]$ codes that are $j$-inadmissible.

By definition any $j$-inadmissible code contains $c_{1}, \ldots, c_{j}$ with the above mentioned property. These $c_{i}$ 's generate a $j$-dimensional vector space since the $c_{i}$ 's are certainly independent: the non-zero coordinates of each $c_{i}$ are unique to that element.

Let $\mathcal{V}$ denote the set of all subsets $\left\{d_{1}, \ldots, d_{j}\right\} \subset C_{2}^{n} \backslash\{0\}$ such that the intersection of the support of $d_{u}$ and $d_{v}$ is empty for all distinct $u, v \in\{1, \ldots, j\}$; thus, in particular, all the $d_{i}$ 's are distinct.

We note that a code $\mathcal{C}$ is $j$-inadmissible if and only if $V \subset \mathcal{C}$ for some $V \in \mathcal{V}$ (this $V$ is not necessarily unique). Moreover Lemma 8 implies that for each $V \in \mathcal{V}$ there are $\left[\begin{array}{c}n-j \\ n-r-j\end{array}\right]$ codes containing $V$; note that if $V \subset \mathcal{C}$ then $\mathcal{C}$ also contains the vector space generated by $V$, which is $j$-dimensional, and apply Lemma 8 . It follows that the total number of $j$-inadmissible codes cannot exceed

$$
|\mathcal{V}|\left[\begin{array}{c}
n-j \\
n-r-j
\end{array}\right] .
$$

In order to estimate $|\mathcal{V}|$ we make the following remark: each element of $\{1, \ldots, n\}$ has to belong to either the support of exactly one of the $d_{i}$ 's or to none (obviously, the information which element of $\{1, \ldots, n\}$ belongs to each of the $d_{i}$ 's uniquely determines the element of $\mathcal{V}$ ). Thus, for each element of $\{1, \ldots, n\}$ there are (at most) $j+1$ possibilities. This readily gives $|\mathcal{V}| \leq(j+1)^{n}$. (We ignore the slight improvements that could be obtained from the fact that the ordering of the $d_{i}$ 's is irrelevant and the supports are non-empty, as they would not affect our estimate).

We therefore infer that the total number of $j$-inadmissible $[n, n-r]$ codes is bounded above by

$$
(j+1)^{n}\left[\begin{array}{c}
n-j \\
n-r-j
\end{array}\right] .
$$

Again by Lemma 8 , it follows that the ratio of the total number of $j$-inadmissible $[n, n-r]$ codes divided by the total number of $[n, n-r]$ codes is bounded above by

$$
\begin{aligned}
\frac{(j+1)^{n}\left[\begin{array}{c}
n-j \\
n-r-j
\end{array}\right]}{\left[\begin{array}{c}
n \\
n-r
\end{array}\right]} & =(j+1)^{n} \prod_{k=n-j+1}^{n} \frac{2^{k-r}-1}{2^{k}-1} \\
& \leq(j+1)^{n} \prod_{k=n-j+1}^{n} \frac{2^{k-r}}{2^{k}} \\
& =(j+1)^{n} 2^{-r j}=2^{n \log _{2}(j+1)-r j} .
\end{aligned}
$$

Here, $\log _{2}$ refers to the logarithm in basis 2 .

Thus, it follows that as soon as $\left(n \log _{2}(j+1)-r j\right)$ is negative, that is

$$
\frac{n}{r}<\frac{j}{\log _{2}(j+1)}
$$

the existence of at least one admissible code is guaranteed. 
From this we deduce (the condition $n \geq r+j$ becomes irrelevant)

$$
D_{j}\left(C_{2}^{r}\right) \geq \log 2 \frac{j}{\log (j+1)} r
$$

as $r$ tends to infinity.

\section{UPPER BOUNDS}

4.1. The crucial lemma. The following lemma is central for our investigations.

Lemma 9. Let $f$ be an asymptotic upper-bounding function. Let $j$ be a positive integer and $p$ be a real number such that one has $\mathrm{D}_{j}\left(C_{2}^{r}\right) \leq p r$ for each sufficiently large $r$. Let finally $c$ denote a solution to the inequality

$$
\frac{p+c-1}{p+c}>f\left(\frac{c}{p+c}\right) \text {. }
$$

Then for each sufficiently large integer $r$, we have

$$
\mathrm{D}_{j+1}\left(C_{2}^{r}\right) \leq(p+c) r .
$$

Proof. By assumption, we have that

$$
\frac{(p+c) r-r}{(p+c) r}=\frac{p+c-1}{p+c}>f\left(\frac{c}{p+c}\right)=f\left(\frac{c r}{(p+c) r}\right) \geq f\left(\frac{c r+1}{(p+c) r}\right),
$$

$f$ being decreasing. If we substitute $\lfloor(p+c) r\rfloor$ for $n$ and $\lfloor c r\rfloor$ for $d$, we obtain (for $r$ sufficiently large and by the continuity of $f$ )

$$
\frac{n-r}{n}>f\left(\frac{d+1}{n}\right)
$$

an equation of the type given in Lemma 6 and we may therefore deduce that

$$
\mathrm{s}_{\leq\lfloor c r\rfloor}\left(C_{2}^{r}\right) \leq n=\lfloor(p+c) r\rfloor \leq(p+c) r .
$$

This now implies, by (3) and using our assumption, that

$$
\begin{aligned}
\mathrm{D}_{j+1}\left(C_{2}^{r}\right) & \leq \min _{i \in \mathbb{N}} \max \left\{p r+i, \mathrm{~s}_{\leq i}\left(C_{2}^{r}\right)-1\right\} \\
& \leq \max \left\{p r+\lfloor c r\rfloor, \mathrm{s}_{\leq\lfloor c r\rfloor}\left(C_{2}^{r}\right)-1\right\} \\
& \leq \max \{(p+c) r,(p+c) r-1\}=(p+c) r,
\end{aligned}
$$

as wanted.

4.2. The upper bounds in Theorem 1, To obtain a proof of these upper bounds, we use the approach described in Section 2 in combination with a bound on the parameters of linear codes originally due to McEliece, Rodemich, Rumsey, and Welch [22, that we recall here (see e.g. 21]): Let us define $h$ to be the binary entropy function, that is (for $0 \leq u \leq 1$ ),

$$
h(u)=-u \log _{2} u-(1-u) \log _{2}(1-u)
$$

and $g(u)=h((1-\sqrt{1-u}) / 2)$. Then the function $f$ equal to

$$
f_{1}(\delta)= \begin{cases}\min _{0 \leq u \leq 1-2 \delta}\left(1+g\left(u^{2}\right)-g\left(u^{2}+2 \delta u+2 \delta\right)\right) & \text { if } \delta \leq 1 / 2 \\ 0 & \text { otherwise }\end{cases}
$$

is an asymptotically upper-bounding function and we may thus apply Lemma 9 . 
We define a sequence $\left(u_{j}\right)_{j \in \mathbb{N}}$ recursively. We set $u_{1}=1$ and, for $j \geq 1$, let $u_{j+1}$ be defined as the solution (if it exists, which is always the case in practice, this solution has to be unique) to the equation

$$
1-\frac{1}{U_{j}+u_{j+1}}=f_{1}\left(\frac{u_{j+1}}{U_{j}+u_{j+1}}\right)
$$

where we define $U_{j}=u_{1}+\cdots+u_{j}$, the sum of the $j$ first values of the sequence $\left(u_{j}\right)_{j \in \mathbb{N}}$. The sequence $\left(U_{j}\right)_{j \in \mathbb{N}}$ corresponds to the coefficient in the upper bound of Theorem $1\left(U_{1}=1, U_{2}=1.395 \ldots, U_{3}=1.770 \ldots, \ldots\right)$, which therefore follows by a repeated application of Lemma 9 .

We point out that for our problem it is actually useful to use this bound, as opposed to bounds whose numerical evaluation is simpler, since for our problem we encounter $\delta$ in a fairly wide range. A simpler strategy, regarding computations, which is - in view of classical results on these bounds - obviously worse, though only slightly so, would be to use another bound proved in 22] (see also 21]), that is the function

$$
f_{2}(\delta)= \begin{cases}h(1 / 2-\sqrt{\delta(1-\delta)}) & \text { if } \delta \leq 1 / 2 \\ 0 & \text { otherwise }\end{cases}
$$

for the first few values, namely 2,3,4 (for 2 this yields the identical bound, yet a slightly weaker one for 3,4 ) where $\delta$ is still fairly large, and then to switch to using a bound that is better for small $\delta$ such as the Elias-Bassalygo bound (see [2] and [21]), that is

$$
f_{3}(\delta)= \begin{cases}1-h((1-\sqrt{1-2 \delta}) / 2) & \text { if } \delta \leq 1 / 2 \\ 0 & \text { otherwise }\end{cases}
$$

with $h$ as above (this is the case $u=0$ of (4.2)). Using this approach, we would for instance get the values 1.776 for $j=3,2.147$ for $j=4,2.512$ for $j=5$ and 4.172 for $j=10$ that is, slightly but noticeably weaker bounds.

4.3. The upper bound in Theorem 2, For the asymptotics we use the method described in Section 2 in combination with the Hamming bound, that is the function $f_{4}$ defined by

$$
f_{4}(\delta)=1-h\left(\frac{\delta}{2}\right) .
$$

We use this bound as the resulting analytic expressions and asymptotic calculations are simpler than for stronger bounds. On the other hand, asymptotically, using say Elias-Bassalygo would not yield a better result. Interestingly, for this particular choice of upper-bounding function, a very elementary proof (cf. below) of the conclusion of Lemma 9 can be obtained with additive means, avoiding the coding theoretic argument that this function is an upper-bounding function. This makes our proof of Theorem 2 essentially self-contained.

Proof. We start with a sequence $S=g_{1}, \ldots, g_{n}$ in $C_{2}^{r}$, where $n=\lfloor(p+c) r\rfloor$. The set $\{1, \ldots, n\}$ has

$$
\sum_{j=0}^{\lfloor c r / 2\rfloor}\left(\begin{array}{c}
\lfloor(p+c) r\rfloor \\
j
\end{array}\right)>\left(\begin{array}{c}
\lfloor(p+c) r\rfloor \\
\lfloor c r / 2\rfloor
\end{array}\right)
$$


subsets of size at most $c r / 2$. Since by assumption

$$
(p+c) h\left(\frac{c}{2(p+c)}\right)>1
$$

and since, as $r$ tends to infinity, $\left(\begin{array}{c}\alpha r \\ \beta r\end{array}\right) \sim c 2^{\alpha h(\beta / \alpha) r}$ (for some constant $c$ depending on $\alpha$ and $\beta$ ), it follows that, for sufficiently large $r$, the number of such sets exceeds $2^{r}$. This implies that there exist two distinct subsets I, J of $\{1, \ldots, n\}$ such that

$$
\sum_{i \in \mathrm{I}} g_{i}=\sum_{i \in \mathrm{J}} g_{i}
$$

This yields

$$
\sum_{i \in \mathrm{I} \triangle \mathrm{J}} g_{i}=0,
$$

- where I $\triangle \mathrm{J}$ denotes the symmetric difference of $\mathrm{I}$ and $\mathrm{J}-$, that is a non-empty zero-sum sequence of length at most $\mathrm{cr}$.

Using the upper-bounding function $f_{4}$, we build a sequence, let us call it $\left(v_{j}\right)_{j \in \mathbb{N}}$ such that $v_{1}=1$ and

$$
\frac{1}{V_{j}+v_{j+1}}=h\left(\frac{v_{j+1}}{2\left(V_{j}+v_{j+1}\right)}\right),
$$

where $V_{j}=v_{1}+\cdots+v_{j}$ for any integer $j \geq 1$. It can be checked easily that such a sequence is well-defined and that for any $j \geq 1$, one has $v_{j} \leq 1$. Then, rewriting (44) as

$$
1-\frac{1}{V_{j}+v_{j+1}}=f_{4}\left(\frac{v_{j+1}}{V_{j}+v_{j+1}}\right),
$$

we may repeatedly apply Lemma 9 and obtain the inequality

$$
\mathrm{D}_{j}\left(C_{2}^{r}\right) \leq V_{j} r
$$

for each integer $j$ and all sufficiently large $r$ (relative to $j$ ).

By the lower bounds in Theorem 2 proved in the preceding Section, we already know that $V_{j}$ tends to infinity when $j$ tends to infinity since

$$
V_{j} \gtrsim \frac{D_{j}\left(C_{2}^{r}\right)}{r} \gg \frac{j}{\log j}
$$

as $r$ tends to infinity ; while $v_{j}$ remains bounded by 1 .

We can now develop (44) to obtain the desired asymptotics. At the first order when $j$ tends to infinity we obtain

$$
\frac{1}{V_{j}+v_{j+1}}=-\frac{v_{j+1}}{2\left(V_{j}+v_{j+1}\right)} \log _{2}\left(\frac{v_{j+1}}{2\left(V_{j}+v_{j+1}\right)}\right)+O\left(\frac{v_{j+1}}{V_{j}+v_{j+1}}\right)
$$

or equivalently

$$
\begin{aligned}
\frac{2 \log 2}{v_{j+1}} & =-\log \left(\frac{v_{j+1}}{2\left(V_{j}+v_{j+1}\right)}\right)+O(1) \\
& =\log \left(V_{j}+v_{j+1}\right)-\log v_{j+1}+O(1) \\
& =\log V_{j}-\log v_{j+1}+O(1)
\end{aligned}
$$


and therefore

$$
\frac{2 \log 2}{v_{j+1}}+\log v_{j+1}=\log V_{j}+O(1) .
$$

It follows that, as $j$ tends to infinity,

$$
v_{j+1} \sim \frac{2 \log 2}{\log V_{j}} .
$$

By (5), we obtain

$$
V_{j+1}-V_{j}=v_{j+1} \lesssim \frac{2 \log 2}{\log j}
$$

and therefore, summing all these estimates yields

$$
V_{j} \lesssim 2 \log 2 \sum_{k=1}^{j-1} \frac{1}{\log k} \sim 2 \log 2 \frac{j}{\log j}
$$

from which the upper bound of Theorem 2 follows.

\section{Heuristics}

In this section, we discuss the quality of the bound in Theorem 1 In investigations on intersecting codes, which are equivalent to determining $\mathrm{D}_{2}\left(C_{2}^{r}\right)$, Cohen and Lempel [5] put forward the heuristic that one can expect that the rate of an intersecting code will not exceed the Gilbert-Varshamov bound

$$
1-h(\delta) \text {. }
$$

Extrapolating this heuristic to the investigation of $\mathrm{D}_{j}\left(C_{2}^{r}\right)$, which might be too optimistic as the restrictions imposed on the code corresponding to extremal example associated to $\mathrm{D}_{j}\left(C_{2}^{r}\right)$ get weaker as $j$ increases, this suggests to use the function $1-h(\delta)$ as if it were an upper-bounding function.

An argument similar to the one in the proof of Theorem 2 thus yields an optimistic heuristic bound $\beta_{j} \lesssim \log 2 \frac{j}{\log j}$.

Regarding heuristic numerical values we get, for example, 1.294 for $j=2,1.550$ for $j=3,1.784$ for $j=4,2.003$ for $j=5$, and 2.984 for $j=10$.

\section{ACKNOWLEDGMENT}

The authors would like to thank G. Cohen for helpful discussions.

\section{REFERENCES}

[1] P. C. Baayen, P. van Emde Boas, Een structuurconstante bepaald voor $C_{2} \oplus C_{2} \oplus C_{2} \oplus C_{2} \oplus C_{6}$, Mathematisch Centrum Amsterdam, WN 27 (1969), 6pp.

[2] L. A. Bassalygo, New upper bounds for error-correcting codes, Problemy Peredači Informacii 1(4) (1965), 41-44.

[3] G. Bhowmik, J.-Ch. Schlage-Puchta, Davenport's constant for groups of the form $\mathbb{Z}_{3} \oplus \mathbb{Z}_{3} \oplus$ $\mathbb{Z}_{3 d}$, pages 307-326, in: Additive Combinatorics (Eds. A. Granville, M.B. Nathanson, and J. Solymosi), CRM Proc. Lecture Notes, 43, Amer. Math. Soc., 2007.

[4] G. Cohen, S. Litsyn, G. Zémor, Binary $B_{2}$-sequences: a new upper bound, J. Combin. Theory Ser. A 94 (2001), 152-155.

[5] G. Cohen, A. Lempel, Linear intersecting codes, Discrete Math. 56 (1985), 35-43.

[6] G. Cohen, G. Zémor, Intersecting codes and independent families, IEEE Trans. Inform. Theory 40 (1994), 1872-1881.

[7] G. Cohen, G. Zémor, Subset sums and coding theory, Structure theory of set addition, pages 327-339, in: Structure Theory of Set Addition (Eds. J.-M. Deshouillers, B. Landreau, and A.A. Yudin), Astérisque 258 (1999), Société Mathémathique de France, 1999. 
[8] Ch. Delorme, O. Ordaz, D. Quiroz, Some remarks on Davenport constant, Discrete Math. 237 (2001), 119-128.

[9] Y. Edel, Sequences in abelian groups $G$ of odd order without zero-sum subsequences of length $\exp (G)$, Des. Codes Cryptogr. 47 (2008), 125-134.

[10] Y. Edel, Ch. Elsholtz, A. Geroldinger, S. Kubertin, L. Rackham, Zero-sum problems in finite abelian groups and affine caps, Q. J. Math. 58 (2007), 159-186.

[11] P. van Emde Boas, A combinatorial problem on finite abelian groups II, Mathematisch Centrum Amsterdam ZW 1969-007 (1969), 60pp.

[12] P. van Emde Boas, D. Kruyswijk, A combinatorial problem on finite abelian groups III, Mathematisch Centrum Amsterdam ZW 1969-008 (1969), 34pp.

[13] M. Freeze, Lengths of factorizations in Dedekind domains, Ph.D. thesis, University of North Carolina at Chapel Hill, 1999.

[14] M. Freeze, W. A. Schmid, Remarks on a generalization of the Davenport constant, manuscript.

[15] W. D. Gao, A. Geroldinger, Zero-sum problems in finite abelian groups: a survey, Expo. Math. 24 (2006), 337-369.

[16] A. Geroldinger, Additive group theory and non-unique factorizations, pages 1-86, in: Combinatorial Number Theory and Additive Group Theory (Eds. A. Geroldinger and I.Z. Ruzsa), Advanced Courses in Mathematics CRM Barcelona, Birkhäuser, 2009.

[17] A. Geroldinger, F. Halter-Koch, Non-Unique Facorizations. Algebraic, Combinatorial and Analytic Theory, Chapman \& Hall, 2006.

[18] A. Geroldinger, R. Schneider, On Davenport's constant, J. Combin. Theory Ser. A 61 (1992), $147-152$.

[19] F. Halter-Koch, A generalization of Davenport's constant and its arithmetical applications, Colloq. Math. 63 (1992), 203-210.

[20] G. Katona, J. Srivastava, Minimal 2-coverings of a finite affine space based on GF(2), J. Statist. Plann. Inference 8 (1983), 375-388.

[21] F. J. MacWilliams, N. J. A. Sloane, The theory of error-correcting codes, North-Holland, 6th ed., 1988.

[22] R. McEliece, E. Rodemich, H. Rumsey, L. Welch, New upper bounds on the rate of a code via the Delsarte-MacWilliams inequalities, IEEE Trans. Information Theory 23 (1977), 157-166.

[23] R. Meshulam, An uncertainty inequality and zero subsums, Discrete Math. 84 (1990), 197200.

[24] D. Miklós, Linear binary codes with intersection properties, Discrete Appl. Math. 9 (1984), $187-196$.

[25] J. E. Olson, A combinatorial problem on finite Abelian groups I, J. Number Theory 1 (1969), $8-10$.

[26] J. E. Olson, A combinatorial problem on finite Abelian groups II, J. Number Theory 1 (1969), 195-199.

[27] A. Potechin, Maximal caps in $A G(6,3)$, Des. Codes Cryptogr., 46 (2008), 243-259.

[28] K. Rogers, A combinatorial problem in Abelian groups, Proc. Camb. Philos. Soc. 59 (1963), $559-562$.

Alain Plagne, Wolfgang A. SChmid

Centre de Mathématiques Laurent Schwartz

UMR $7640 \mathrm{du}$ CNRS

École polytechnique

91128 Palaiseau cedex

France

E-mail address: plagne@math.polytechnique.fr, schmid@math.polytechnique.fr 